Check for updates

Cite this: Phys. Chem. Chem. Phys., 2019, 21, 14429

Received 25th November 2018 Accepted 13th March 2019

DOI: $10.1039 / c 8 c p 07248 e$

rsc.li/pccp

\section{Field modified spin-orbit potential curves of IBr. Preliminary dynamical results $\dagger$}

\begin{abstract}
Cristina Sanz-Sanz (D) ${ }^{a}$ and Graham A. Worth (D) *b
In a seminal work the photodissociation of $\mathrm{IBr}$ has been controlled using a strong non-resonant IR pulse [Sussman et al., Science, 2006, 314, 274], changing the branching ratio of products in different final states via the relative timing of pump and control pulses. In this paper, we revisit the control of this molecule. Potential surfaces for the complete spin-orbit manifold of $\mathrm{IBr}$ states dissociating into the ground and first excited states of the constituent atoms have been calculated at the multi-reference configuration interaction ( $\mathrm{MRCl}$ ) level of theory as a function of applied field. Both the strength and direction of field have been taken into account and it is seen how the avoided crossing between the states thought to be key in the control mechanism shift as a function of field strength. These surfaces will enable full calculations of the molecule in the pump-control field. Preliminary dynamics calculations with the field placed along the molecular axis show that a Hamiltonian including all 36-states agrees with earlier results and is able to model the basic features of the control. However, just like earlier results, this restricted model is not able to reproduce the timescale of the control.
\end{abstract}

\section{Introduction}

The strong-field control of the photodissociation of $\mathrm{IBr}$ by Stolow and co-workers ${ }^{1,2}$ is a classic example of affecting the outcome of a chemical reaction by modifying the potential curves near a curve crossing using the non-resonant dynamic Stark effect. The crossing modified by the pulse appears between two excited spin-orbit states that dissociate to two different dissociation channels. The control pulse separates the states avoiding the dissociation in the lower channel, producing an inversion of the dissociation ratio. Computer simulations using a simple model for the coupled potentials have supported the interpretation of the mechanism, but questions remain. In particular, the timescale for the control is much shorter in the simulations, with the simulations showing that the photodissociation reaction is over in around $100 \mathrm{fs}$ while the experiments show a control effect out to $500 \mathrm{fs}^{1,3}$ To understand the origin of this discrepancy it is first necessary to build a complete and accurate theoretical description of IBr able to reproduce the experimental data. Comparison of this full description and the models used previously will provide the missing link.

\footnotetext{
${ }^{a}$ Departamento de Química Física Aplicada, Módulo 14, Universidad Autónoma de Madrid, 28049 Madrid, Spain. E-mail: cristina.sanz@uam.es; Tel: +34 914973922

${ }^{b}$ Department of Chemistry, University College London, 20 Gordon Street, London WC1H OAJ, UK. E-mail: g.a.worth@ucl.ac.uk

$\dagger$ Electronic supplementary information (ESI) available: Further results from simulations. See DOI: 10.1039/c8cp07248e
}

The IBr system has received much attention in the last three decades mainly because of the crossing excited-states which dissociate to two different dissociation channels. The initial studies, ${ }^{4-6}$ more than 80 years ago, were focused on the interpretation of the absorption spectrum by vibrational analysis. The first theoretical studies, ${ }^{7}$ a decade later, started a series of papers dedicated to the electronic description of the potential energy curves ${ }^{8-11}$ with special attention to the crossing of the excited states. The description of the halogen atoms used relativistic effective core potentials (ECP) including spin-orbit interactions. The description of the dissociation energies using ECP's was deficient, and a later study ${ }^{12}$ used an optimised basis-set to described correctly all the states and crossings of the states dissociating to the ground and first excited spin-orbit states of I and Br atoms. ${ }^{12}$ These optimised basis sets are the basis sets used in this paper. With the first descriptions of the potential energy curves the first spectroscopic theoretical studies also appeared ${ }^{13}$ focusing on the adiabatic and diabatic effects of the excited states crossings.

The first photodissociation study ${ }^{13}$ used several coupling regimes to explain the predominance of excited state of $\mathrm{Br}$ in the photodissociation products. The application of laser pulses provided several extra studies in vibration-rotation excitation, ${ }^{14}$ wavepacket absorption spectra, ${ }^{15,16}$ and wavepacket predissociation. ${ }^{17}$ In addition to the use of the laser pulse as a method to promote the system from lower to excited vibro-rotational or electronic states, pulses were used to control the photofragmentation of IBr. The first studies to control the photodissociation used coherent control ${ }^{18,19}$ and optimal control theory. ${ }^{20}$ The dynamic 
Stark control experiments mentioned above, in which an intermediate strength control pulse is used after the excitation to shift the curves involved in the crossing, appeared one decade later: first with a theoretical study using a three-state model $^{1}$ and a year later, the experimental evidence ${ }^{2}$ of the curve shifting of the excited electronic states controlling the photodissociation channel. The influence of the parameters of the control pulse (width, intensity, duration of the pulse and delay of the control pulse) using a three-states model with calculated potential curves, dipole moments and polarizabilities was subsequently published. $^{3}$

The theoretical simulation of a time-resolved spectroscopic study takes place in two-steps. First, potential surfaces and couplings between them must be calculated using $a b$ initio quantum chemistry calculations. Then, quantum dynamics simulations solving the time-dependent Schrödinger equation is performed including the light pulse and obtaining the measured signal, in this case the branching ratio of products as a function of control pulse delay time. Most simulations including a light pulse are in the weak-field limit, adding the pulse as an extra time-dependent term in the Hamiltonian. In this case the field is in the Stark-shift intermediate regime and is strong enough to shift the potential surfaces, but not strong enough to lead to ionisation. To model this, the potential curves can be computed adding a static electric field in the $a b$ initio calculations and potential energy curves produced that depend on the electric field strength and the orientation angles in which the field is applied. The potential energy curves for the diatomic IBr are then three dimensional surfaces depending on inter-atomic distance, field strength and orientation angle. The light pulses can then be modeled as a time dependent function of field intensity, modifying the potentials accordingly. This is an extension of the electric-nuclear Born-Oppenheimer approximation of Balint-Kurti and co-workers. ${ }^{21}$

The electric-field dependent potential calculations are computationally challenging. The number of distinct states dissociating to $\operatorname{Br}\left({ }^{2} \mathrm{P}\right)$ and $\mathrm{I}\left({ }^{2} \mathrm{P}\right)$ when spin-orbit (SO) coupling is not considered is a twelve. We refer to these pure spin states as spin-free states. The atoms of $\mathrm{Br}$ and I have, however, strong SO coupling, being $3685 \mathrm{~cm}^{-1}$ for $\mathrm{Br}$ and $7603 \mathrm{~cm}^{-1}$ for I. These values are not negligible and if they are ignored no curve crossing is found between excited states. The ground state of halogen atoms is ${ }^{2} \mathrm{P}$, which splits to ${ }^{2} \mathrm{P}_{3 / 2}$ and ${ }^{2} \mathrm{P}_{1 / 2}$ when SO is considered, giving rise to four dissociation channels for long inter-atomic distances. When SO coupling is included the total number of distinct mixed-spin states rises to 23 . We refer to these as spin-orbit, or SO states. A scheme of the SO splitting defining the dissociation asymptotes is given in Fig. 1.

The details of the ab initio calculations are given in Section 2.1. The calculation of the SO coupling unfortunately provides a problem, as random phases in the state wavefunctions lead to sign changes and swapping of states between neighbouring points on the PES, even when each point is computed using the reference wavefunction of the previous point. These discontinuities have to be corrected to produce smooth curves for the energies, dipole moments and SO couplings. The calculation of the potential curves

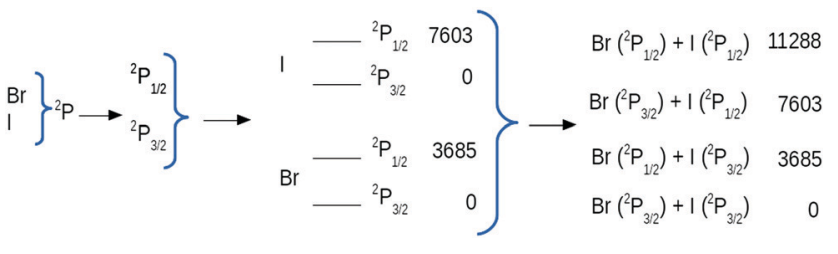

Fig. 1 Spin-orbit splitting (in $\mathrm{cm}^{-1}$ ) of the ground states of $\mathrm{Br}$ and I which produce four possible dissociation channels. Experimental data. ${ }^{22}$

affected by an external field complicates the smoothing process, which otherwise could be easily made by computing the overlap between two consecutive points. The method that we use to create smooth curves will be presented in Section 2.2 of this paper. The PE curves obtained are described in Section 3.1.

The aim of the computation of the potential curves under the influence of an static electric field, is the simulation of the experiments $^{2}$ of the dynamic Stark control of the photodissociation of IBr. In Section 3.2 of this paper we present preliminary results of the dynamical simulation of the photodissociation of $\mathrm{IBr}$, using a control pulse oriented along the intermolecular axis of the molecule, considering all the electronic states. The light field is also coupled to the molecule using only the dipoles and polarisabilities rather than the full 3D field-dependent surfaces. The final section, Section 4, presents the conclusions.

\section{Methods}

\subsection{Ab initio calculations}

The states required for the simulation of the control of the photodissociation of IBr must include all those dissociating to the four SO states of the ground states of I and Br. For the computation of these states, the MOLPRO quantum chemistry package $^{23}$ was used. Because MOLPRO can only compute SO couplings from the configuration interaction (CI) wavefunction, all the electronic structure results are computed at CI level of calculation.

A first attempt using pseudopotentials in both atoms failed, probably due to the large number of states needed. An extensive study of the 23 SO states of IBr was presented in 2006 by Patchkovskii ${ }^{12}$ using basis sets optimised for I and $\mathrm{Br}$ to better describe the full set of SO states dissociating to the I + Br channels. The author provided us with the optimised basis sets and all our calculations are made using these which are relativistic Gaussian basis sets adapted to the third-order Douglas-Kroll Hamiltonian derived from ref. 24 . The resulting contraction of the basis sets are (21s 16p 11d 2f)/[9s 7p 5d 2f] for Br and (24s 20p 14d 2f)/[10s 8p $6 \mathrm{~d} 2 \mathrm{f}$ ] for I. A more detailed explanation about the basis set optimisation is presented in the original paper. ${ }^{12}$

The active space used for our calculations included a total of $33 \mathrm{~A}^{\prime}$ and $13 \mathrm{~A}^{\prime \prime}$ orbitals using $C_{\mathrm{s}}$ symmetry, from which $28 \mathrm{~A}^{\prime}$ and $11 \mathrm{~A}^{\prime \prime}$ orbitals remained closed. This active space accommodates 10 active electrons in a total of 7 orbitals. The number of singlet and triplet states from both symmetries which dissociate to the ground states of $\mathrm{I}$ and $\mathrm{Br}$ are $5^{1} \mathrm{~A}^{\prime}, 5^{1} \mathrm{~A}^{\prime \prime}, 4^{3} \mathrm{~A}^{\prime}$ and $4^{3} \mathrm{~A}^{\prime \prime}$. The Multi-Configurational Self Consistent Field (MCSCF) provides 
Table 1 Spin-orbit splitting (in $\mathrm{cm}^{-1}$ ) of free I and $\mathrm{Br}$ atoms. Comparison with previous calculations and experiments

\begin{tabular}{lll}
\hline & $\mathrm{Br}$ & $\mathrm{I}$ \\
\hline Exp. $^{22}$ & 3685 & 7603 \\
Previous calc. $^{12}$ & 3459 & 7043 \\
This work & 3526 & 8046
\end{tabular}

the set of 18 potential curves when the SO couplings are not considered, all degenerate at long distances, dissociating to the ground electronic states of I and Br. The MCSCF wavefunction was then used as reference for the CI calculations, which provide better potential curves, and allow the calculation of the SO couplings. The same active space was considered for the $\mathrm{CI}$ calculations and the optimised wavefunction saved and used in the SO matrix calculation. Due to the symmetry adopted, $C_{\mathrm{s}}$, the SO matrix is composed of 36 states as the $\Pi$ and $\Delta$ states appear in both irreducible representations. With respect to the $\Sigma$ states the + states appear in the $\mathrm{A}^{\prime}$ representation and the states appear in the $\mathrm{A}^{\prime \prime}$. The calculation of the SO matrix needed to include all the states dissociating to the ground states of I and $\mathrm{Br}$, because otherwise the SO states do not give the correct four dissociation channels.

Patchkovskii used this basis set with a similar active space and the GAMESS package. ${ }^{12}$ As our calculations are obtained using MOLPRO, we present the comparison of the SO splitting for free I and $\mathrm{Br}$ in Table 1. The SO splittings are of the same order, but the values obtained using MOLPRO have smaller differences from the experimental values. The spin-orbit splitting for $\mathrm{Br}$ is underestimated by $159 \mathrm{~cm}^{-1}\left(226 \mathrm{~cm}^{-1}\right.$ in the previous calculations) and is overestimated for I by $443 \mathrm{~cm}^{-1}$ (underestimated by $570 \mathrm{~cm}^{-1}$ in previous calculations).

\subsection{Fitting of the field-coupled potentials}

For grid-based quantum dynamics simulations, the potential energy curves under the effect of the electric field need to be provided as a global function. This can either be in the form of an analytical function, or using an interpolation method to provide a description of the curves without the effort of fitting to an analytical function. Considering that in this system we have to describe the potential surfaces of 36 states, all their couplings, and the full dipole moment matrix, an automatic spline interpolation procedure is the obvious option. Furthermore, the interpolation gives a good description of the properties of this system because the potential curves vary smoothly with variation of the electric field strength and molecule orientation. A standard 3-dimensional spline interpolation was used for the description of all electronic properties in this paper.

For the quantum dynamics simulations, we require diagonal electronic properties (potential energy and dipole moments) and off-diagonal elements (transition dipole moments and spin-orbit couplings) in the basis of the spin-free states, in which the kinetic energy operator can be taken to be diagonal, all under the influence of an electric field. It is well known that quantum calculations do not maintain the relative phase of the wavefunction from one point to the next, so that off-diagonal properties can have changes of sign.
In addition, states that are degenerate or very close to each other can appear in the wrong order and states can swap along a coordinate. Therefore, the first step before we can fit any of these properties is to produce smooth curves.

The correction of the sudden changes in off-diagonal matrix elements can be obtained, in principle, using the overlap between two consecutive points, but with the electric field on the overlaps were often not evidently near to 1 or -1 , i.e. mixing occurred. Even in the field free case the off-diagonal elements mixed significantly and were not smooth functions of the nuclear distance. Thus, we developed a procedure that makes use of the fact that transformation between the spin-free and spin-orbit bases is local and is not affected by the changes of the relative phase of the wavefunctions. At each value of the bond length, $R$, the spin-free potential matrix, $\mathbf{W}$, is related to the spin-orbit potentials in the diagonal matrix, $\mathbf{V}$ by a unitary transformation

$$
\mathbf{W}(R)=\mathbf{U}^{\dagger} \mathbf{V}(R) \mathbf{U}
$$

The diagonal elements of the field-free matrices $\mathbf{W}^{(0)}$ and $\mathbf{V}^{(0)}$ are obtained from quantum chemistry calculations and the task is to define the off-diagonal elements of $\mathbf{W}$. For this, the following optimisation scheme was used

1. An initial point was chosen at a long internuclear distance in which the properties are well defined. Here the full $\mathbf{W}^{(0)}$ matrix is taken from quantum chemistry calculations.

2. Move to the next internuclear distance (shorter bond), and take the off-diagonal elements of $\mathbf{W}^{(0)}$ from the previous point as a guess.

3. The off-diagonal terms are optimised using a conjugate gradient optimiser to ensure the eigenvalues of $\mathbf{W}^{(0)}$ are identical to the corresponding values of $\mathbf{V}^{(0)}$.

4. The optimised values are used as the reference for the next point.

This procedure thus gives functions of both the non-adiabatic and spin-orbit coupling elements, assuming that they change slowly as the bond length changes. It ensures a field-free spin-free potential matrix with the correct spin-orbit eigenvalues.

A slightly modified version of this procedure was then used to provide the off-diagonal elements of the potential matrix as a function of field-strength. Starting with the optimised field-free potential matrix at a particular bond length, $\mathbf{W}^{(0)}(R)$, a guess for the off-diagonal elements at field strength $E$ was taken as

$$
\mathbf{W}(R, E)=\mathbf{W}^{(0)}(R)+\mathbf{D}(R, E)
$$

where $\mathbf{D}(E)$ is the field-free dipole matrix in the spin-free representation multiplied by the field strength, $\mathbf{D}(E)=-\mathbf{E} \cdot \mathbf{D}^{(0)}$. I.e. step 2 is changed to

2. At each inter-atomic distance, move to the next field strength, and sum the potential matrix (W) elements with $\mathbf{D}(E)$, the field-free dipole moment matrix multiplied by the field strength.

This initial guess matrix was then optimised so that the eigenvalues of the optimised $\mathbf{W}(R, E)$ are constructed to be the spin-orbit eigenvalues at that field strength.

In order to see if the procedure has worked the optimised Hamiltonian matrix eigenvalues can be compared with the 

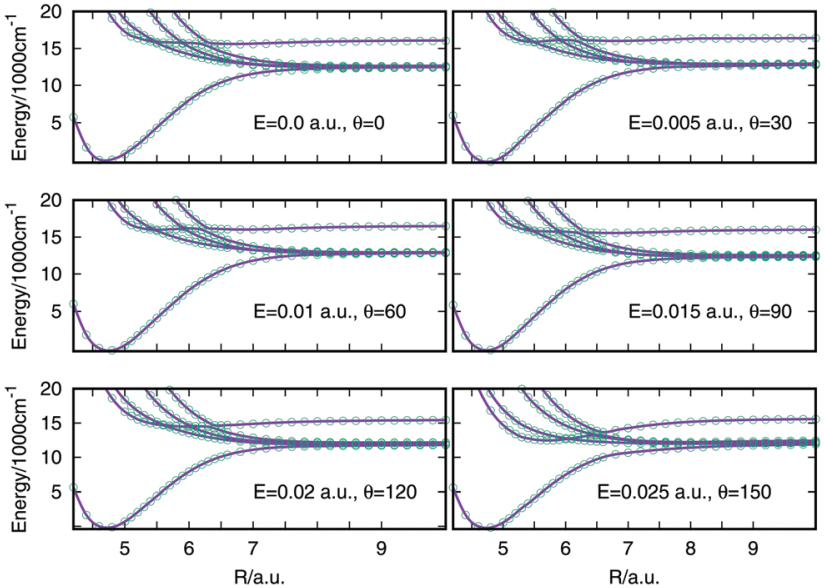

Fig. 2 Comparison between the spin-orbit coupled states (eigenvalues of the spin-orbit matrix) obtained from the optimised procedure (empty circles) and the spin-orbit coupled states obtained from MOLPRO (lines). The states plotted are $1^{1} \Sigma_{0}^{+}, 1^{3} \Pi_{0+}, 1^{1} \Pi_{1}, 2^{3} \Pi_{1}, 2^{1} \Pi_{1}$.

eigenvalues obtained from MOLPRO for each $R, E$ and $\theta$ values. In Fig. 2 this comparison is shown for a selection of cuts through the surfaces, where the optimised points are plotted with green empty circles and the eigenvalues from MOLPRO are the purple lines. The field strength and angle of the electric field are specified in each plot. For every field strength and angle selected, the lines pass through all the points, evidence that the optimisation procedure provides the same eigenvalues. The optimised off-diagonal elements are smooth and can be spline interpolated to be used in the quantum dynamics calculations.

\subsection{Dynamics calculations}

After calculating the potential energy surfaces, the system dynamics is obtained by solving the time-dependent Schrödinger equation using numerically exact wavepacket propagation as implemented in the QUANTICS ${ }^{25}$ set of programs.

The grid used for all propagations presented in this paper is a Fast Fourier Transform (FFT) collocation, with a total of 256 points running from interatomic distances between 4.0 and 12.00 Bohr. The propagation used an Adam-Bashforth-Moulton predictor corrector (ABM) integration scheme. To avoid the use of long grids the wavepacket is absorbed before it reaches the end of the grid using a complex absorbing potential (CAP). The CAP has the form $-i W(Q)=-i \eta\left(Q-Q_{\mathrm{c}}\right)^{b} \theta\left(Q-Q_{\mathrm{c}}\right)$, where $\theta(x)$ denotes the Heaviside's step function and $Q_{\mathrm{c}}$ is the point where the CAP is switched on. The optimised CAP used in this study has an exponent set to $b=2$, a strength $\eta=0.00116$ Hartree and is placed at $R=10.0$ Bohr. The study of the branching ratio requires the study of the portion of the wavepacket that reaches this CAP, assuming that at this point the dissociation has occurred. QUANTICS provides the flux through the absorbing potential in either representation, spin-orbit coupled and non-coupled.

The initial wavepacket was formed using energy relaxation, propagating an initial guess in imaginary time until convergence is reached. The imaginary propagation used the complete set of states and couplings so the initial propagation starts with a non-negligible population of the wavepacket in some of the spin-free excited states, i.e. the ground-state function includes a mix of both singlet and triplet character.

\section{Results}

\subsection{Potential energy curves}

The experiment of the control of the photodissociation of $\mathrm{IBr}$ is based on modifying the potential energy curves near an avoided crossing between states dissociating to two different spin-orbit states of $\mathrm{Br}$ atom. The system is first excited to an energy region above the first spin-orbit excited state $\left(\mathrm{I}\left({ }^{3} \mathrm{P}_{3 / 2}\right)+\operatorname{Br}\left({ }^{3} \mathrm{P}_{1 / 2}\right)\right)$. It then evolves and reaches the avoided crossing where it has the possibility to dissociate in either channel. The control is produced by a pulse, non-resonant with the states near the avoided crossing, so that no excitation is produced within the states, but the curves are modified so that the system is forced to dissociate in the spin-orbit excited state. The theoretical complication that arises when spin-orbit coupling is included is that all states dissociating to the ground electronic states of I and $\mathrm{Br}$ have to be considered in the calculations and the spinorbit matrix needs all states and all couplings to produce the correct spin-orbit eigenvalues.

The total number of distinct states dissociating to the ground states of $\mathrm{I}$ and $\mathrm{Br}$ are 12 and these states split into 23 states when spin-orbit is included: $\left({ }^{1} \Sigma_{0-}^{-},{ }^{1} \Sigma_{0+}^{+}(\times 2),{ }^{1} \Pi_{1}(\times 2)\right.$, ${ }^{1} \Delta_{2},{ }^{3} \Sigma_{0+}^{-},{ }^{3} \Sigma_{1}^{-},{ }^{3} \Sigma_{0-}^{+}(\times 2),{ }^{3} \Sigma_{1}^{+}(\times 2),{ }^{3} \Pi_{1}(\times 2),{ }^{3} \Pi_{0_{+}}(\times 2),{ }^{3} \Pi_{0-}(\times 2)$, ${ }^{3} \Pi_{2}(\times 2),{ }^{3} \Delta_{2},{ }^{3} \Delta_{1}$ and $\left.{ }^{3} \Delta_{3}\right)$. Ten of these states correlate to the ground state atoms $\left({ }^{2} \mathrm{P}_{3 / 2}\right)$, five states each correlate to the $\mathrm{SO}$ excited state to one of the atoms (5 states to $\operatorname{Br}\left({ }^{2} \mathrm{P}_{1 / 2}\right)+\mathrm{I}\left({ }^{2} \mathrm{P}_{3 / 2}\right)$ and 5 states to $\left.\operatorname{Br}\left({ }^{2} \mathrm{P}_{3 / 2}\right)+\mathrm{I}\left({ }^{2} \mathrm{P}_{1 / 2}\right)\right)$ and the last three correlate to both atoms in their $\mathrm{SO}$ excited state.

In Table 2 the spin free (uncoupled) and the spin-orbit coupled states are listed, sorted in increasing order of energy of the spin-free states. The table presents the labeling of states for both representations and the energy $\left(\mathrm{in}^{-1}\right.$ ) at the equilibrium distance (Frank-Condon point) of the ground state. The zero of energies considered for the values in this table is at the dissociation energy of the spin-free states. The labeling of the states is obtained using the eigenvectors of the full matrix and the expectation value of the $L_{z}{ }^{2}$, both provided by MOLPRO. Because our calculations are made using $C_{\mathrm{s}}$ symmetry, the labeling of the states is tedious but is reliable since we can simultaneously use the expectation value of $L_{z}{ }^{2}$ and the fact that spin-orbit coupling components appear in different irreducible representation. Most states in the spin-orbit representation have a clear major component and can be related to a spin-free state. Two states, however, have a mixed character and are composed of approximately equal amounts of the $1^{1} \Delta_{2}$ and $1^{3} \Delta_{2}$ spin-free states. The colours denote the SO states dissociating to the four different channels. The avoided crossing controlled to produce the dissociation in the first excited channel is created by $1^{3} \Pi_{0+}$ and $1^{3} \Sigma_{0+}^{-}$. By symmetry, these two states are not coupled by non-adiabatic coupling and the avoided crossing is due to spinorbit coupling of about $120 \mathrm{~cm}^{-1}$. 
Table 2 Electronic energies (in $\mathrm{cm}^{-1}$ ) at Frank-Condon distance sorted in increasing order of the non-coupled states (spin-free states). The two first columns present the adiabatic states, the third and fourth columns the spin-orbit coupled states, and the final column the dissociation channel, numbered from the lowest energy

\begin{tabular}{|c|c|c|c|c|}
\hline \multicolumn{2}{|c|}{ Spin-free states } & \multicolumn{3}{|c|}{$\underline{\text { Spin-orbit states }}$} \\
\hline Symbol & Energy & Symbol & Energy & Channel \\
\hline $1^{1} \Sigma^{+}$ & -15750 & ${ }^{1} \Sigma_{0+}^{+}$ & -15750 & 1 \\
\hline \multirow[t]{4}{*}{$1^{3} \Pi$} & 3456 & ${ }^{3} \Pi_{2}$ & 1463 & 1 \\
\hline & & ${ }^{3} \Pi_{1}$ & 2781 & 1 \\
\hline & & ${ }^{3} \Pi_{0-}$ & 5846 & 1 \\
\hline & & ${ }^{3} \Pi_{0+}$ & 6607 & 1 \\
\hline $1^{1} \Pi$ & 8324 & ${ }^{1} \Pi_{1}$ & 9188 & 1 \\
\hline \multirow{4}{*}{$2^{3} \Pi$} & 22428 & ${ }^{3} \Pi_{2}$ & 21156 & 1 \\
\hline & & ${ }^{3} \Pi_{1}$ & 21986 & 1 \\
\hline & & ${ }^{3} \Pi_{0-}$ & 24390 & 1 \\
\hline & & ${ }^{3} \Pi_{0+}$ & 24696 & 1 \\
\hline $2{ }^{1} \Pi$ & 25450 & ${ }^{1} \Pi_{1}$ & 26320 & 2 \\
\hline \multirow[t]{2}{*}{$1^{3} \Sigma^{-}$} & 28151 & ${ }^{3} \Sigma_{0+}^{-1}$ & 26512 & 2 \\
\hline & & ${ }^{3} \Sigma_{1}^{-}$ & 29016 & 2 \\
\hline \multirow[t]{2}{*}{$1^{3} \Sigma^{+}$} & 29143 & ${ }^{3} \Sigma_{0-}^{+}$ & 30285 & 2 \\
\hline & & ${ }^{3} \Sigma_{1}^{+}$ & 30471 & 2 \\
\hline $1^{1} \Delta$ & 31424 & ${ }^{1} \Delta_{2}+1^{3} \Delta_{2}$ & 32010 & 3 \\
\hline $1^{1} \Sigma^{-}$ & 33939 & ${ }^{1} \Sigma_{0-}^{-}$ & 36580 & 4 \\
\hline $2^{1} \Sigma^{+}$ & 39238 & ${ }^{1} \Sigma_{0+}^{+}$ & 37287 & 3 \\
\hline \multirow[t]{3}{*}{$1^{3} \Delta$} & 39965 & ${ }^{3} \Delta_{3}$ & 36651 & 3 \\
\hline & & ${ }^{3} \Delta_{2}+1^{1} \Delta_{2}$ & 41040 & 3 \\
\hline & & ${ }^{3} \Delta_{1}$ & 44929 & 4 \\
\hline \multirow[t]{2}{*}{$2^{3} \Sigma^{+}$} & 40618 & ${ }^{3} \Sigma_{1}^{+}$ & 41605 & 3 \\
\hline & & ${ }^{3} \Sigma_{0-}^{+}$ & 44909 & 4 \\
\hline
\end{tabular}

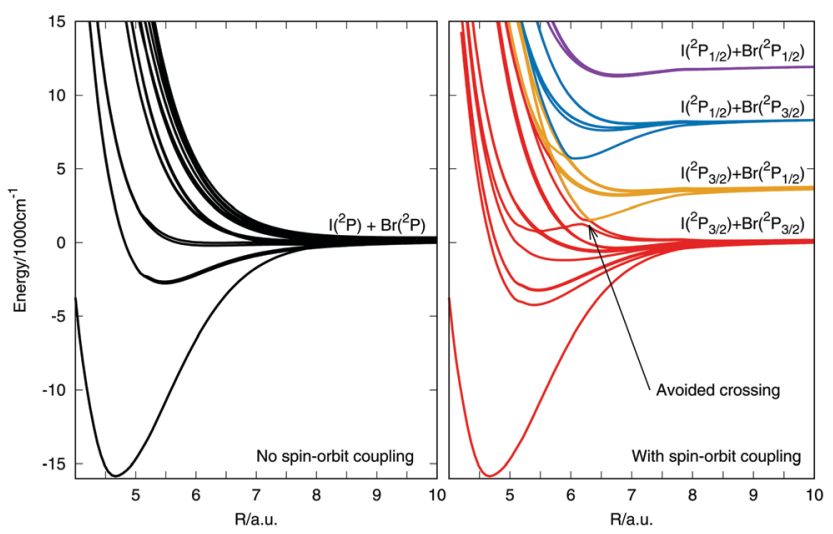

Fig. 3 Potential energy curves of the $\mathrm{IBr}$ system. The left panel presents the 12 spin-free states dissociating to the ground states of I and $\mathrm{Br}$. The right panel shows the 23 states when the spin-orbit coupling is considered.

The potential energy curves are presented in Fig. 3. The left panel shows the 12 spin-free states. They are all degenerate for long internuclear distances and dissociate to $\mathrm{I}$ and $\mathrm{Br}$ in their ground electronic state. The right panel shows the 23 states dissociating to the spin-orbit electronic states of I and $\operatorname{Br}\left({ }^{3} \mathrm{P}_{3 / 2}\right.$ and $\left.{ }^{3} \mathrm{P}_{1 / 2}\right)$. The states dissociate to four channels, which can be related to the states in Table 2 by the given channel number. The avoided crossing is highlighted by a black arrow. An expanded version of the potential curves including spin-orbit coupling is provide in Fig. S1 of the ESI, $\dagger$ along with the assignment of the states at the Franck-Condon point. It should be noted that the curves differ slightly from those presented in our earlier paper ref. 3 . The earlier potentials were calculated at the same level of theory but used a constant value of $150 \mathrm{~cm}^{-1}$ for the spin-orbit coupling in contrast to the complete coordinate dependent coupling used here.

To assess the quality of the new potential surfaces, a comparison with known experimental and calculated data is given in Table 3. Experimental data are taken from high resolution spectroscopy studies. ${ }^{27-31}$ In addition, Vrakking and coworkers ${ }^{26}$ performed pump-probe experiments on IBr using a range of experimental excitation energies from $547 \mathrm{~nm}\left(18282 \mathrm{~cm}^{-1}\right)$ to $590 \mathrm{~nm}$ $\left(16949 \mathrm{~cm}^{-1}\right)$, corresponding to the first excited dissociation channel and the curve crossing, respectively. In our calculations the values obtained are $18999 \mathrm{~cm}^{-1}$ for the first excited dissociation channel and $16781 \mathrm{~cm}^{-1}$ for the curves crossing point. Both values are in good agreements with the experimental values.

A comparison has also been made to the detailed theoretical analysis of the potential surfaces made by Li et al. ${ }^{32}$ using multireference configuration interaction (MRCI) based on a 10 electron, 11 orbital active space and a natural orbital basis set. A plot of our spin-coupled surfaces compared to their surfaces is given in Fig. S2 of the ESI $\dagger$ for the states with total angular momentum $\Omega=0^{+}$and $\Omega=0^{-}$, in which the crossings occur. The surfaces have same shape, but in our calculations the avoided crossings have wider gaps and the minima are deeper.

The reaction we are studying is started by an excitation pulse promoting the system to the excited states with energies near $20000 \mathrm{~cm}^{-1}$. All the states in this energy region dissociate to the lowest dissociation channel, but the avoided crossing connects one state to the first excited channel, allowing branching of a dissociating wavepacket. The control pulse aims to modify the curves so that when the system reaches the avoided crossing region the curves separate and dissociation in the first excited channel is enhanced.

By including a static field in the quantum chemistry calculations, the potential energy curves were also obtained as a function of the field strength and the orientation of the electric field with respect to the molecular axis. The internuclear distances in the computation of the potential energy curves were varied from 4.0 to 10 a.u. in intervals of 0.2 a.u., except from 5.0 to 6.6 where the intervals were

Table 3 Computed and experimental spectroscopic constants

\begin{tabular}{|c|c|c|c|}
\hline & & $R_{\mathrm{e}}(\AA)$ & $T_{\mathrm{e}}\left(\mathrm{cm}^{-1}\right.$ \\
\hline $\mathrm{X}^{1} \Sigma_{0+}^{+}$ & $\begin{array}{l}\text { This work } \\
\text { Exp. }^{33} \\
\text { Calc. }^{32}\end{array}$ & $\begin{array}{l}2.4692 \\
2.479078 \\
2.482\end{array}$ & \\
\hline $1^{3} \Pi_{2}$ & $\begin{array}{l}\text { This work } \\
\text { Exp. }^{27} \\
\text { Calc. }^{32}\end{array}$ & $\begin{array}{l}2.851 \\
2.857 \\
2.863\end{array}$ & $\begin{array}{l}11195 \\
11190 \\
11454\end{array}$ \\
\hline $1^{3} \Pi_{1}$ & $\begin{array}{l}\text { This work } \\
\text { Exp. }^{31} \\
\text { Calc. }^{32}\end{array}$ & $\begin{array}{l}2.894 \\
2.8583 \\
2.899\end{array}$ & $\begin{array}{l}12175 \\
12369 \\
12473\end{array}$ \\
\hline $\mathrm{B}^{3} \Pi_{0}^{+}$ & $\begin{array}{l}\text { This work } \\
\text { Exp. }^{28} \\
\text { Calc. }^{32}\end{array}$ & $\begin{array}{l}2.915 \\
2.83 \\
2.898\end{array}$ & $\begin{array}{l}16211 \\
16168 \\
16507\end{array}$ \\
\hline
\end{tabular}



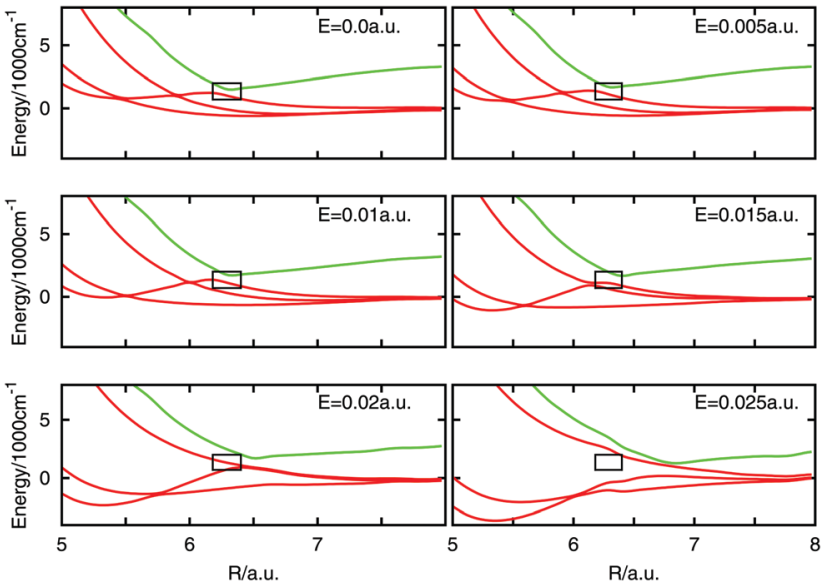

Fig. 4 Potential energy curves for different values of the electric field strength for $\theta=0$ of some states near the avoided crossing. The black box frame the position of the avoided crossing.

reduced to 0.1 a.u., to describe better the Frank Condon region. The field strength was varied from 0.005 to 0.025 a.u. in intervals of 0.005 a.u. at different angles of orientation with respect to the axis of the molecule. The molecule lies along the $Z$ axis with $\mathrm{Br}$ in the positive direction and the electric field is oriented in the $Y Z$ plane. The electric field components are defined as $\varepsilon_{y}=E \times \sin \theta$ and $\varepsilon_{z}=E \times \cos \theta$, where $E$ is the field strength and $\theta$ is the angle formed between the molecular axis and the electric field vector.

The electric field distorts the potential energy and the effect on the curves near the avoided crossing is shown in Fig. 4. All the panels present the effect of the application of the electric field parallel to the internuclear axis and the field strength is specified in each panel. A black box frames the avoided crossing. The curves get visually distorted and the states producing the crossing separate from each other. At a field strength of 0.025 a.u. a marked shift in the curves is seen which is probably due to errors in the quantum chemistry at this high field. The effect in the potential curves of the angle between the molecular axis and the electric field vector is presented in Fig. 5 for an electric field strength of 0.02 a.u. The black box is maintained in place to mark the position of the field-free avoided crossing. At $0^{\circ}$ the avoided crossing with an electric field of 0.02 a.u. is moved to longer distances and the minimum becomes deeper. The minimum disappears as the angle increases but is seen to return for larger angles approaching $180^{\circ}$.

\subsection{Dynamical calculations}

The best evidence that all the states, couplings and dipole moment elements are accurate is their use in quantum dynamics calculations to simulate experimental results. In this paper we present preliminary results, similar in spirit to the earlier threestate model study, ${ }^{3}$ but this time using the full set of states and couplings involved in the system. This means that rather than using the surfaces as a function of field strength and angle, the field-free potential curves and couplings are used with dipole and polarisability matrices to couple the system to the field.
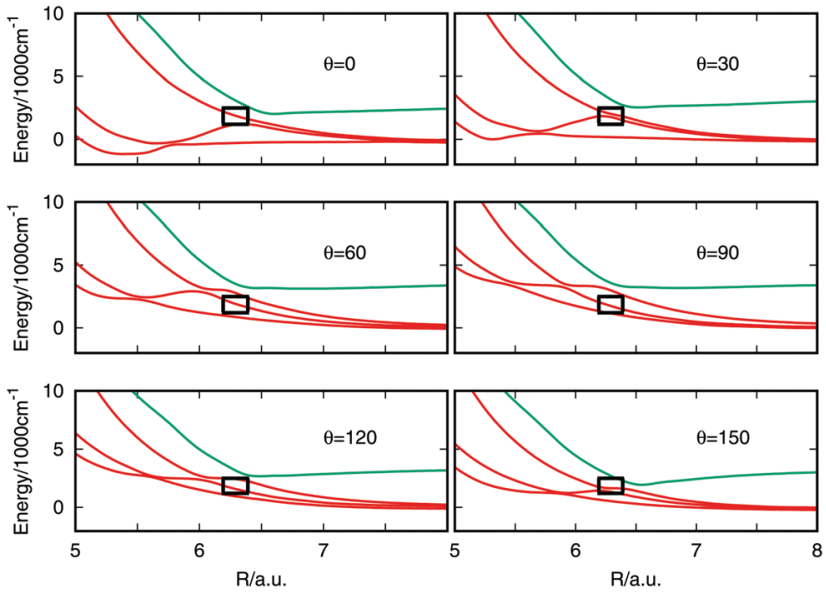

Fig. 5 Potential energy curves for different values of the angle between the electric field vector and the molecular axis of some states near the avoided crossing. The field strength is fixed in 0.02 a.u.

The Hamiltonian used for the time propagation of the system is thus written as follows

$$
\hat{H}_{j k}=\hat{T} \delta_{j k}+W_{j k}^{(0)}(R)+V_{j k}^{\mathrm{eff}}(R, E, t)
$$

where $V_{j k}^{\text {eff }}=V_{j k}^{d}+V_{j k}^{S}$ and the elements $V_{j k}^{d}$ and $V_{j k}^{S}$ are

$$
\begin{gathered}
V_{j k}^{d}(R, E, t)=-\mathbf{d}_{j k}(R) \cdot \mathbf{e}\left(E_{1}(t) \cos \left(\omega_{1} t\right)+E_{2}(t) \cos \left(\omega_{2} t\right)\right) \\
V_{j k}^{S}(E, t)=-\frac{1}{4} \alpha_{j k}\left(E_{1}(t)^{2} \cos ^{2}\left(\omega_{1} t\right)+E_{2}(t)^{2} \cos ^{2}\left(\omega_{2} t\right)\right)
\end{gathered}
$$

where the pairs of subscripts denote that the quantities are matrix elements. $W_{j k}^{(0)}(R)$ is the field-free potential matrix with potential energy in the diagonal elements and spin-orbit couplings in the off-diagonal. The oscillating field is composed of two pulses: an excitation and a control. These are denoted with the subscripts 1 and 2 and each have a time-dependent envelope $E(t)$ with polarisation direction $\boldsymbol{e}$ and a central frequency $\omega$. It should be noted that the frequencies of the two pulses are very different, with the excitation pulse lying in the UV and the control pulse in the IR.

$V_{j k}^{d}(R, E, t)$ is the multiplication of the dipole matrix elements $\left(\mathbf{d}_{j k}(R)\right)$ with the field envelope and oscillating function in the direction of the field. $V_{j k}^{S}(E, t)$ is the Stark control term, i.e., the interaction of the polarizability with the square of the electric field. This term modifies the potential curves following the envelope of the pulse. The dipole matrix, $x, y, z$ components and modulus, are obtained from MOLPRO calculations.

For these preliminary calculations we only use the $z$ component of the transition dipole moment, considering this component as parallel to the internuclear axis. For light polarisation along the $z$-axis, only the $2^{1} \Sigma_{0}^{+}$state is bright, i.e. has a significant transition dipole from the ground-state. All other states will be populated due to higher order couplings to the field, or by intensity borrowing from this state. It is interesting to note that the $1^{1} \Delta_{2}$ has such high spin-orbit coupling with the $1^{3} \Delta_{2}$ that they cannot be separated in the spin-orbit states. Thus the triplet manifold can be directly accessed from the ground-state. 


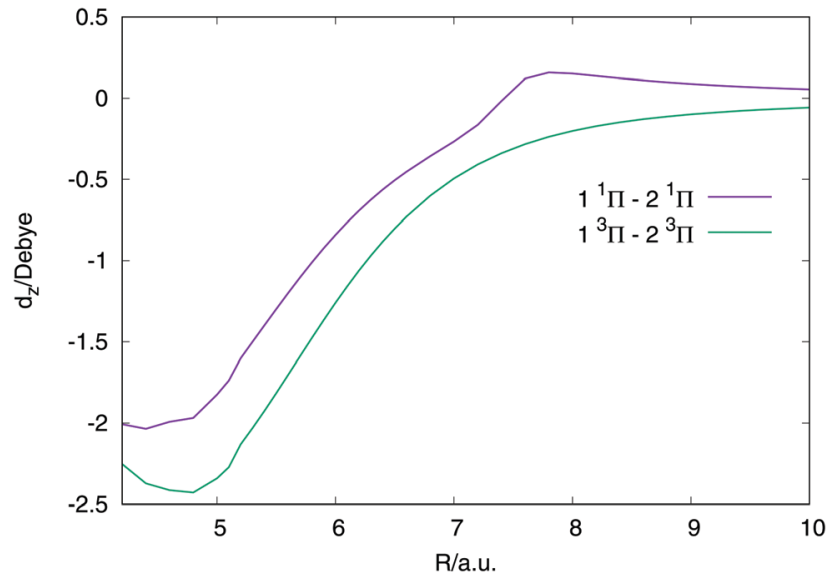

Fig. 6 The $z$ component of the two largest transition dipole moments between the states, as specified in the plot. All other transition dipoles are lower that 0.2 Debyes along all the values of $R$.

In these first simulations, the polarizability is kept fixed in value. It is calculated from the derivative of the dipole moment, $d_{z}$ with respect to an electric field parallel to the internuclear axis at the internuclear distance where the avoided crossing is, $R=6.5$ a.u. This is the reason why in eqn (5) the polarizability is not dependent on internuclear distance but the dipole moments and transition dipole moments are. In Fig. 6 the two biggest transition dipole moments are shown along the internuclear distance. The rest of transition dipole moments are lower than 0.2 Debyes for all distances. In Table 4 the constant polarizabilities used in the dynamical calculations are listed.

In order to understand the natural behaviour of the wavepacket, the vertical excitation to each of the excited states was analysed. After exciting the ground state wavepacket to each

Table 4 Polarizability calculated at the distance of the avoided crossing ( $R=6.5$ a.u.) as the derivative of the dipole moment for each electronic state. 1 a.u. $=0.529^{3} \AA^{3}$

\begin{tabular}{l} 
Polarizability ( \\
\hline $1^{1} \Sigma_{0}^{+}=-95.1$ \\
$1^{1} \Pi_{1}=-103.5$ \\
$2^{1} \Pi_{1}=-48.0$ \\
$1^{1} \Delta_{2}=-68.0$ \\
$2^{1} \Sigma_{0}^{+}=-65.2$ \\
$1^{3} \Pi_{2}=-124.4$ \\
$1^{3} \Pi_{1}$ \\
$1^{3} \Pi_{0}$ \\
$2^{3} \Pi_{2}=-46.4$ \\
$2^{3} \Pi_{1}$ \\
$2^{3} \Pi_{0}$ \\
$1^{3} \Sigma_{1}^{+}=-57.2$ \\
$1^{3} \Sigma_{0}^{+}$ \\
$1^{3} \Delta_{3}=-62.5$ \\
$1^{3} \Delta_{2}$ \\
$1^{3} \Delta_{1}$ \\
$2^{3} \Sigma_{1}^{+}=-62.1$ \\
$2^{3} \Sigma_{0}^{+}$ \\
$1^{1} \Sigma_{0}^{-}=-62.8$ \\
$1^{3} \Sigma_{0}^{-}=-74.9$ \\
$1^{3} \Sigma_{1}^{-}$
\end{tabular}
Polarizability $\left(\alpha_{j k}\right) /$ a.u.

$1^{1} \Sigma_{0}^{+}-1^{1} \Delta_{2}=0.3$

$1^{1} \Sigma_{0}^{+}-2^{1} \Sigma_{0}^{+}=1.0$

$1^{1} \Pi_{1}-2{ }^{1} \Pi_{1}=9.7$

$1^{1} \Delta_{2}-2^{1} \Sigma_{0}^{+}=0.6$

$1^{3} \Pi_{2}-2^{3} \Pi_{2}=8.7$

\section{$1^{3} \Pi_{1}$}

$2^{3} \Pi_{1}$

$2^{3} \Pi_{0}$

$1^{3} \Sigma_{1}^{+}=-57.2$

$1^{3} \Sigma_{0}^{+}$

$1^{3} \Delta_{3}=-62.5$

$1^{3} \Delta_{2}$

$1^{3} \Delta$

$2^{3} \Sigma_{1}^{+}=-62.1$

$2^{3} \Sigma_{0}^{+}$

$1 \Sigma_{0}^{3} \Sigma_{0}^{-}=-74.9$
$1^{3} \Sigma_{1}^{-}$

$1^{3} \Sigma_{1}^{-}$ spin-free excited state in turn, we conclude that only the excitation to the $m_{\mathrm{s}}$ components, in both symmetries $\mathrm{A}^{\prime}$ and $\mathrm{A}^{\prime \prime}$, of the $1^{1} \Pi$ and $1^{3} \Pi$ can be considered as dissociative excitations. By this we mean that after the excitation, at least $97 \%$ of the wavepacket reaches the dissociation region. In the spin-orbit states we can assign the population to each state and more importantly in this study, to the different dissociation channels. In the ESI $\dagger$ the populations of each spin free state, summed over the $z$-components of the total angular $(J=L+S)$, are plotted for the excitation to each of the components of the $1^{3} \Pi$ (Fig. S3, ESI $\dagger$ ) and $1^{1} \Pi$ (Fig. S4, ESI $\dagger$ ). For all propagations the population in the initially excited state starts decreasing after $50 \mathrm{fs}$, therefore it seems that this is the time that it takes for the wavepacket to reach the crossing region.

Due to the many states and spin-orbit couplings a detailed study of the state populations is tedious and not very illuminating. However, the strong SO coupling between the $1^{1} \Pi$ and $1^{3} \Pi$ is noticeable, with fast oscillatory population transfer between these states seen in many of the simulations. The complicated details can be exemplified by looking at the populations after starting in the two components of the ${ }^{1} \Pi$ state. The $\mathrm{A}^{\prime}$ component population flows into a number of states, including the ground ${ }^{1} \Sigma^{+}$state. In contrast, the $\mathrm{A}^{\prime \prime}$ component population ends predominantly in the $2^{3} \Pi$ state.

In Fig. 7 the state populations for these excitations are plotted summed up into the IBr dissociation channels of the spin-coupled states. These are listed on the right hand side of Fig. 1 and seen in the potential curves of Fig. 3. There are four, defined with channel 1 as the lowest energy channel. In these figures it is even clearer to see that the first sudden change in population occurs around $50 \mathrm{fs}$, after this time population oscillates for certain time until the wavepacket reaches the dissociation region and it starts being absorbed by the complex absorbing potential (CAP). In both cases the population is initially in channel 1 , but population transfer to channels 2 and 3 occurs. A difference is the time that takes the wavepacket to reach the dissociation region. It seems that the excitation to the ${ }^{1} \Pi_{J}$ states runs slightly faster, with the CAP starting to absorb the wavepacket before $200 \mathrm{fs}$, while for the excitation to the ${ }^{3} \Pi_{J}$ the wavepacket starts being absorbed around $200 \mathrm{fs}$ as well but the absorption in channel 1 is slower and the population decreases to below $10 \%$ after $300 \mathrm{fs}$, instead of $230-250 \mathrm{fs}$ in the other cases.

The flux ending up in the different channels for all the different excitations is given in Table S1 in the ESI. $\dagger$ A representative result for propagation starting in the non-dissociative states is shown for the ${ }^{1} \Sigma_{0-}^{-}$state in Fig. 8 . The state populations, shown summed into the different channels in Fig. 8(a), oscillates along the whole propagation, and the wavepacket never reaches the crossing or the dissociation region. The expectation value of the bond length is shown in Fig. 8(b). It is seen to initially stretch, but the motion is then damped after $100 \mathrm{fs}$, ending at a slightly elongated value compared to the equilibrium bond length. This is a surprising result as the system energy is above the dissociation threshold. It seems that a highly excited electronic wavepacket has formed, with fast population transfer between the states taking the 

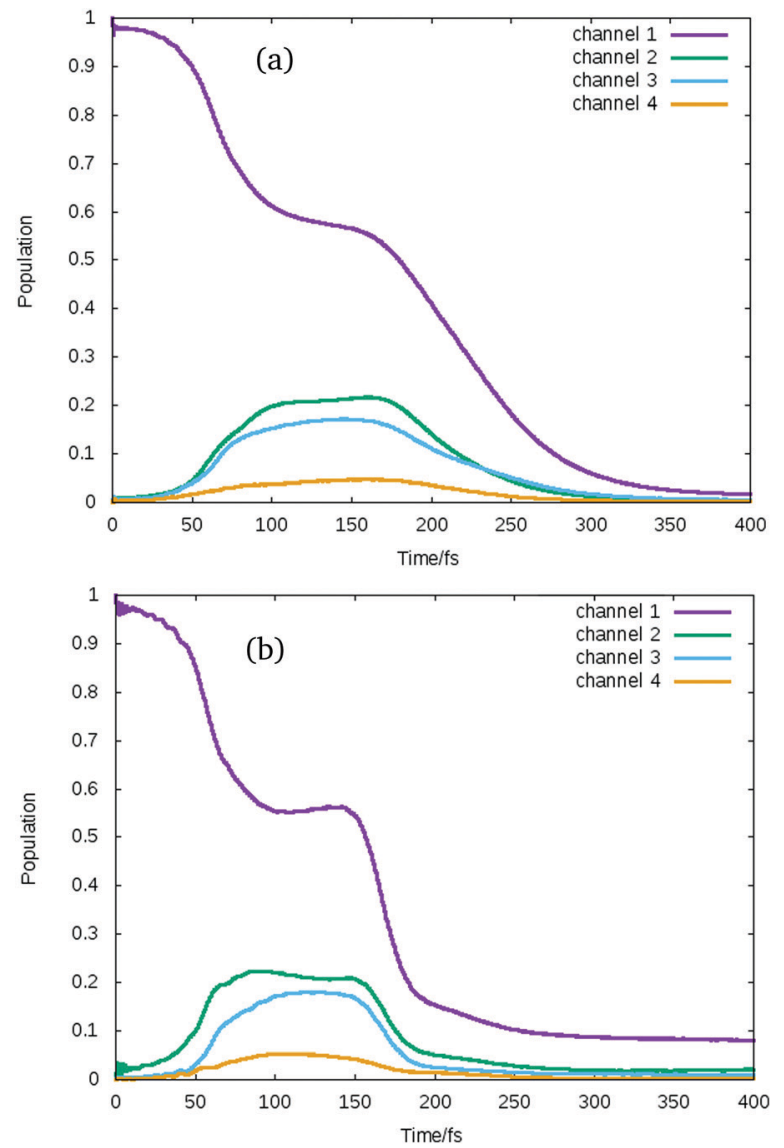

Fig. 7 Average population of spin-orbit coupled states summed up into the four spin-coupled dissociation channels after vertical excitation to all components of (a) $1^{3} \Pi$ and (b) $1^{1} \Pi$.

energy out of the nuclear motion. Further analysis is required to understand this behaviour.

The vertical excitation simulations provide the natural timescale for the IBr photo-dissociation when described by a one-dimensional model. The next step is to simulate the nonresonant Stark shifting excitation, including laser pulses that follow the scheme used in the experiment. Two pulses are used: the first is to promote the system to the excited states, with an energy slightly above the dissociation limit of the two first dissociation channels (100 fs and $520 \mathrm{~nm}$ ), and the second, the control pulse (150 fs, $1.7 \mu \mathrm{m}$ with intensity below $10^{-13} \mathrm{~W} \mathrm{~cm}^{-2}$ ). The control pulse is applied at a series of time delays before, during and after the excitation pulse.

In this way the wavepacket is promoted to the excited state using a pump pulse, shown by the purple curves in the upper panels of Fig. 9 and 10. The control of the dissociation channel is produced using the non-resonant Stark effect, which theoretically does not interact with the dipole moment, so that the shift of the potential curves follows the electric field given by the envelope of the pulse. For this reason we employ two different kinds of control pulses, one which oscillates with a certain frequency, green line in the upper panel of Fig. 9 and one which is only the Gaussian pulse envelope, green line in the upper panel of Fig. 10. The branching ratio is then obtained by calculating the flux going into each
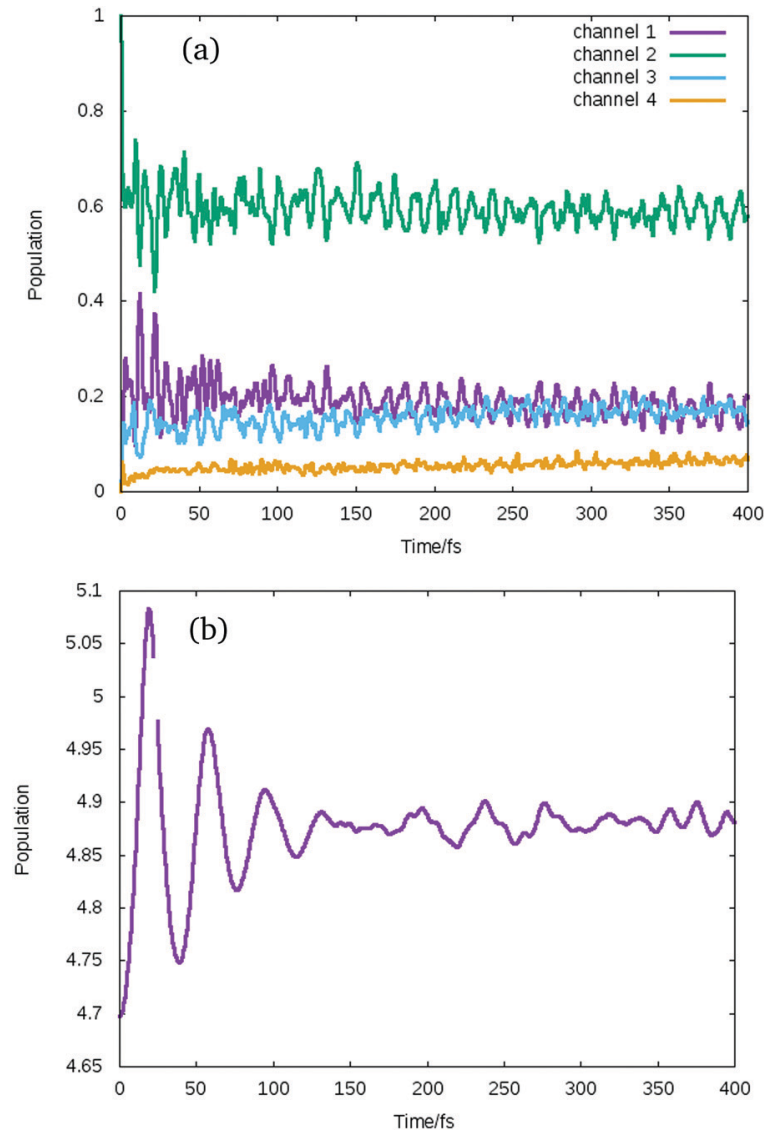

Fig. 8 (a) Average population of spin-orbit coupled states sum up over all the states dissociating to each dissociation channel when the wavepacket is excited to the high lying dark state $\left(1^{1} \Sigma^{-}\right)$. (b) The expectation value of the bond length after the excitation.

dissociation channel. With just the excitation pulse (i.e. no control pulse applied), only $36.21 \%$ of the wavepacket reaches the dissociation channels after 1650 fs. Of this, $99.42 \%$ is the lowest energy channel with $\mathrm{I}$ and $\mathrm{Br}$ in their ground-states, and $0.57 \%$ is in the next channel with $\mathrm{Br}$ in its excited-state. A negligible amount exits in the upper channels.

The change in branching ratio of $\mathrm{IBr}$ in the two lower dissociation channels obtained using the two pulses is shown in the lower panels of Fig. 9 and 10. This is defined as the fractional change relative to the branching ratio calculated without a control pulse being applied. Negative values of the ratio means that the dissociation into the $\operatorname{Br}\left({ }^{2} \mathrm{P}_{3 / 2}\right)+\mathrm{I}\left({ }^{2} \mathrm{P}_{3 / 2}\right)$ channel is enhanced (lowest dissociation channel), and positive values of the change in branching ratios means that the dissociation into the $\operatorname{Br}\left({ }^{2} \mathrm{P}_{1 / 2}\right)+\mathrm{I}\left({ }^{2} \mathrm{P}_{3 / 2}\right)$ channel is enhanced (first excited dissociation channel). The purple dot-line curve is the theoretical 3-state model change in branching ratio, and the purple dashed curve the experimental result from Sussman et al. ${ }^{2}$ The other two curves, in light blue and orange are the change in branching ratio of our simulation using the 36 potential energy states and the $z$-component of the transition dipole moments between all the states with non-negligible values. The difference between the light blue and orange curve is the interaction of the control pulse. 

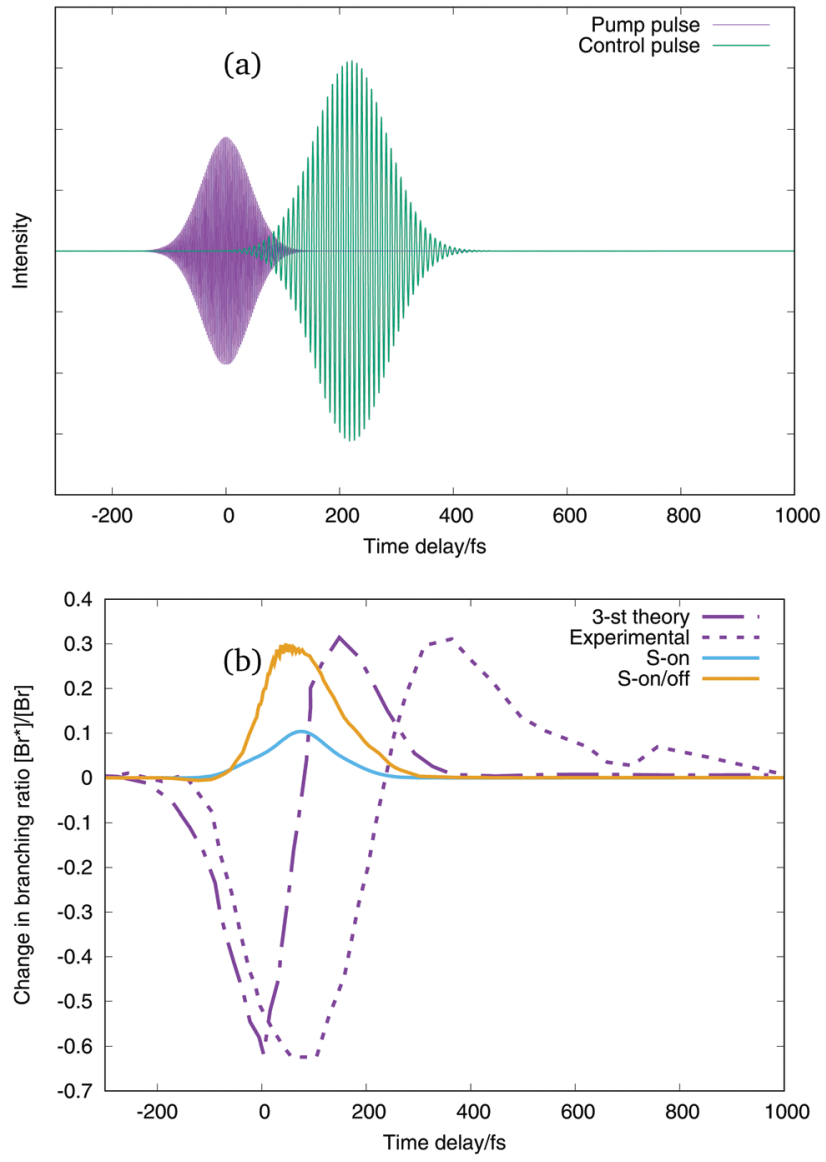

Fig. 9 (a) Excitation and control pulses for a time delay of the control pulse of $220 \mathrm{fs}$. (b) Change in branching ratio $\mathrm{Br} / \mathrm{Br}$ with respect to the time delay of the control pulse. Purple dot-line curve: three-state theoretical simulation, ${ }^{2}$ purple dashed-line curve: experimental values ratio, ${ }^{2}$ light blue line: our results when control pulse only interacts with on-diagonal potential energy term, orange line: our results when control pulse interacts with on and off diagonal potential energy terms.

The light blue curve presents the change in branching ratio when both control pulses only interacts with the on-diagonal terms of the dipole matrix and polarisabilities (on-diagonal interaction), and the orange curve presents the change in branching ratio when the control pulse interacts with the on and the off-diagonal terms of the dipole matrix and polarizabilities (on/off-diagonal interaction), see eqn (4) and (5). It is worth pointing out here that the effect of the control pulse comes from the interaction with the fixed dipole moments and polarizabilities. The simulations in this work do not include the dependency of the potential energy curves with the field strength.

Considering first the results shown in Fig. 9, the on-diagonal interaction and the on/off-diagonal interaction give very similar results: in both cases only the dissociation in the $\mathrm{Br}^{*}$ channel is significantly enhanced. The on-diagonal interaction, light blue line, enhancement of the $\mathrm{Br}^{*}$ channel is however, lower than the full on/off-diagonal interaction. The orange line shows a very little enhancement of the $\mathrm{Br}$ channel for small negative time delays. In both simulations the enhancement of the excited dissociation channel does not agree with either the theoretical
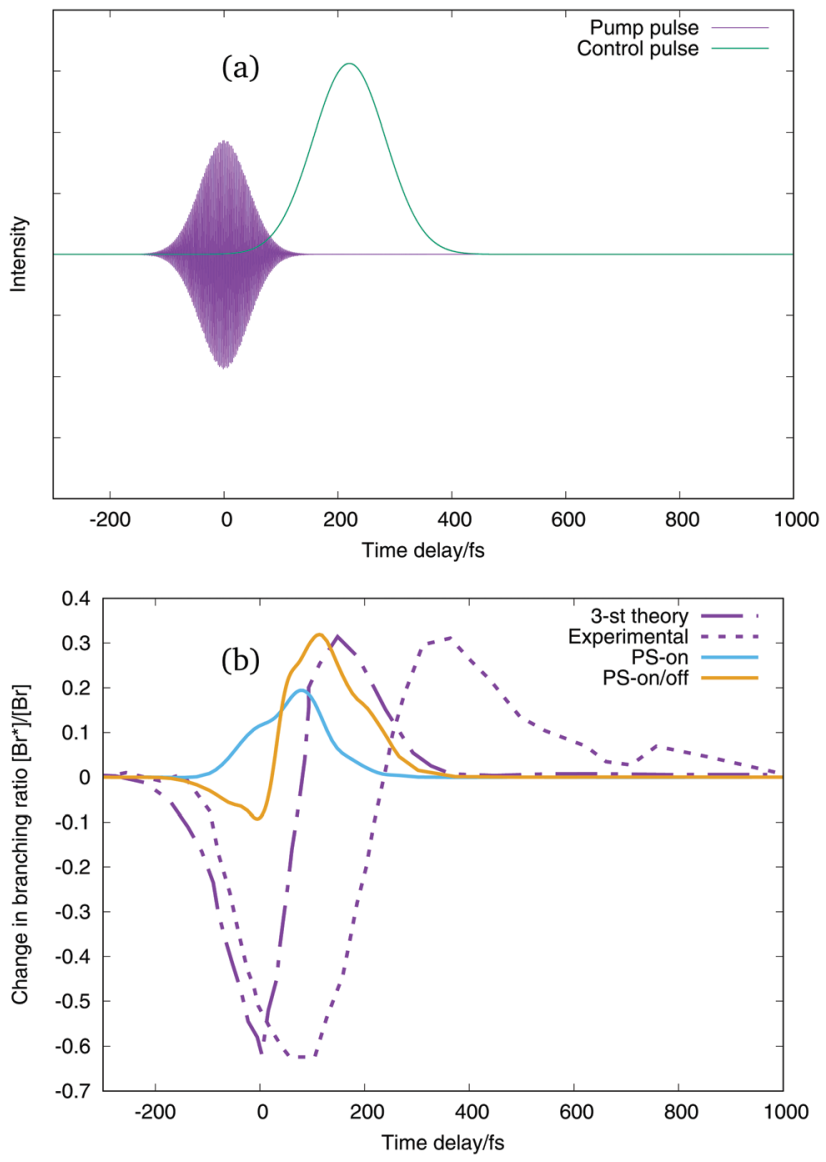

Fig. 10 (a) Excitation pulse and the envelope control pulse for a time delay of the control pulse of $220 \mathrm{fs}$. (b) Change in branching ratio $\mathrm{Br} / \mathrm{Br}$ with respect to the time delay of the control pulse. Purple dot-line curve: three-state theoretical simulation, ${ }^{2}$ purple dashed-line curve: experimental values ratio, ${ }^{2}$ light blue line: our results when control pulse only interacts with on-diagonal potential energy term, orange line: our results when control pulse interacts with on and off diagonal potential energy terms.

3-state (purple dot-line curve) or experimental (purple dashed curve) results with the enhancement of the $\mathrm{Br}^{*}$ channel appearing at shorter times delays.

In Fig. 10 the change in branching ratio calculated when the control pulse consists only of a Gaussian envelope are shown. Again, both the on-diagonal interaction and on/off diagonal interaction calculations show a significant enhancement of the $\mathrm{Br}^{*}$ channel. Now, the change in branching ratio for the on/offdiagonal interaction behaves like the 3 -state model simulation, ${ }^{2}$ with an initial enhancement of the $\mathrm{Br}$ channel before the $\mathrm{Br}^{*}$ enhancement. It is, however, much lower in our results than either the earlier model or experiment. Probably the fact that in our results the enhancement of the lower dissociation channel is smaller could come from the resonant interaction of the pump pulse with the dipole moment. The laser excitation in our simulation can populate higher excited states which could produce dissociation in higher channels $\left(\mathrm{Br}+\mathrm{I}^{*}\right.$ and $\left.\mathrm{Br}^{*}+\mathrm{I}^{*}\right)$. It can also be noted that the result from the new 36-state model has more structure in the branching ratio profile than the simple 3-state model. This is in line with our earlier study using 
an extended 3-state model that identified other control mechanisms to be present in addition to the moving of the avoided crossing. These included enhancement to the lower channel due to the changed excitation process at $t=0$ fs due to the overlapping excitation and control pulses. ${ }^{3}$

The 3-state model simulation and the on/off-diagonal pulsepotential interaction of the Gaussian envelope thus present the same behaviour, with an enhancement of the lower dissociation channel, Br, when the delay of the control pulse is at short negative times, and an enhancement of the excited dissociation channel, $\mathrm{Br}^{*}$, for positive values of time delays. It is evident, however, that none of the theoretical simulations have the same time scales as the experimental results. The $\mathrm{Br}$ channel peak in the simulations peaks at around 0 fs and the $\mathrm{Br}^{*}$ channel peak around $100 \mathrm{fs}$, whereas in the experiment the first peak is around $100 \mathrm{fs}$ and the second $350 \mathrm{fs}$.

\section{Conclusions}

In this work are presented the calculations of the potential energy curves of all the states dissociating in the ground state of IBr. This includes the calculations of the spin-orbit couplings within all states which dissociate into the four spin-orbit states of $\mathrm{I}\left({ }^{2} \mathrm{P}_{3 / 2},{ }^{2} \mathrm{P}_{1 / 2}\right)$ and $\operatorname{Br}\left({ }^{2} \mathrm{P}_{3 / 2},{ }^{2} \mathrm{P}_{1 / 2}\right)$. The full set of potential curves and spin-orbit couplings are calculated under the influence of several electric-field strengths and angle orientations. The field strength runs from 0 to 0.025 a.u. and the angle orientation from 0 to 180 degrees. A procedure to smooth the spin-orbit couplings along the intermolecular distance is presented and probed to provide the right results after the diagonalisation of the spin-orbit matrix. The three-dimensional curves $(r, E, \theta)$ are fitted using spline interpolation.

Preliminary results of dynamical calculations using the whole set of states are also presented. The wavepacket propagation runs in the field-free potential curves and is excited and controlled using the pulse-dipole and pulse-polarisability interactions. It is found that the results obtained for the change in dissociation branching ratio follow the same behaviour as the previous 3-states theoretical results as well as the experimental results as long as all interactions are included and the oscillating field in the control pulse is ignored. The dissociation into the lowest channel is mainly enhanced when the pump and control pulses starts to overlap in time. Looking at the potential curves as a function of field strength, this can be explained by the fact that the control pulse separates the curves before the excitation, causing a decreasing on the excitation into states above the first excited dissociation channel. However, when the control pulse is applied at times delays in which the wavepacket is approaching the crossing, the separation of the states helps the dissociation into the first excited channel.

The propagation time scale seen in the simulations is, however, still far from the experimental result and the relative magnitude of the branching ratio peaks is incorrect. The time scale is a particular issue, seen in all simulations to date. In the experiments the first, negative, peak in the change in branching ratio occurs around 100 fs and the second, positive, peak around 350 fs. In contrast, the simulations provide the first peak at 0 fs and the second at 100 fs. These differences must arise from the approximations made in the simulations. In line with earlier work, the model is only one-dimensional, with the molecule oriented along the $z$-axis. In fact, the main difference in the present model compared to the earlier 3-state model is the addition of more states. In this fixed orientation the excited molecule has an intrinsically fast dynamics: the avoided crossing is reached within $50 \mathrm{fs}$ and the molecule has fully dissociated by $200 \mathrm{fs}$ making it a mystery as to how in the experiments control is seen up to $500 \mathrm{fs}$. In addition to this fixed geometry, again following earlier work, only the transition dipole along the $z$-axis and a static polarisability were included as these usually provide the dominant interaction with the field.

The next step will be to include the full interaction of the field with all the states and all the transition dipole moments to see the effect of the angle dependency and strength. The present simulations indicate that it must be the interplay of all factors that slows the observed dynamics significantly and will lead to a full understanding of the experimental data.

\section{Conflicts of interest}

There are no conflicts to declare.

\section{Acknowledgements}

This paper is dedicated to Mike Ashfold on the occasion of his 65th birthday in recognition of his pioneering work in timeresolved spectroscopy and molecular reaction dynamics.

\section{References}

1 B. J. Sussman, M. Y. Ivanov and A. Stolow, Phys. Rev. A: At., Mol., Opt. Phys., 2005, 71, 051401.

2 B. J. Sussman, D. Townsend, M. Y. Ivanov and A. Stolow, Science, 2006, 314, 278-281.

3 C. Sanz-Sanz, G. W. Richings and G. A. Worth, Faraday Discuss., 2011, 153, 275-291.

4 H. Cordes, Z. Phys., 1932, 74, 34.

5 W. Brown and G. E. Gibson, Phys. Rev., 1932, 40, 529.

6 W. Brown, Phys. Rev., 1932, 42, 355.

7 R. S. Mulliken, Phys. Rev., 1942, 61, 277.

8 S. Y. Lee and Y. S. Lee, Chem. Phys., 1991, 187, 302.

9 W. A. Jong, J. Styszynski, L. Visscher and W. C. Nieuwpoort, J. Chem. Phys., 1998, 108, 5177.

10 J. Pittner and P. Jungwirth, Chem. Phys. Lett., 2000, 321, 281. 11 H. S. Lee, W. K. Cho, Y. J. Choi and Y. S. Lee, Chem. Phys., 2005, 311, 121.

12 S. Patchkovskii, Phys. Chem. Chem. Phys., 2006, 8, 926-940. 13 M. S. Child, Mol. Phys., 1976, 32, 1495.

14 S. C. Leasure, K. F. Milfeld and R. E. Wyatt, J. Chem. Phys., 1981, 74, 6197.

15 M. Shapiro and H. Bony, J. Chem. Phys., 1985, 83, 1588. 
16 H. Guo, J. Chem. Phys., 1993, 99, 1685.

17 A. N. Hussain and G. Roberts, J. Chem. Phys., 1999, 110, 2474.

18 I. Levy, M. Shapiro and P. Brumer, J. Chem. Phys., 1990, 93, 2493.

19 C. K. Chan, P. Brumer and M. Shapiro, J. Chem. Phys., 1991, 94, 2688.

20 P. Gross, D. B. Bairagi, M. K. Misha and H. Rabitz, Chem. Phys. Lett., 1994, 223, 263.

21 S. Zou, G. G. Balint-Kurti and F. R. Manby, J. Chem. Phys., 2007, 127, 44107.

22 NIST Atomic Spectra Database, NIST Standard Reference Database 78.

23 H.-J. Werner, P. J. Knowles, G. Knizia, M. F. R. Manby, et al., MOLPRO, version 2008.1, a package of ab initio programs, 2008.

24 T. Tsuchiya, M. Abe, T. Nakajima and K. Hirao, J. Chem. Phys., 2001, 115, 4463-4472.
25 G. A. Worth, K. Giri, G. W. Richings, I. Burghardt, M. H. Beck, A. Jäckle and H.-D. Meyer, QUANTICS, a suite of programs for molecular QUANTum dynamICS simulations, Version 1.1, 2015.

26 M. Vrakking and D. M. V. A. Stolow, J. Chem. Phys., 1996, 106, 5647-5650.

27 D. R. T. Appadoo, P. F. Bernath and J. L. Roy, Can. J. Phys., 1994, 21, 1265-1272.

28 L. E. Selin and B. Soderborg, Ark. Fys., 1962, 21, 515.

29 T. Yukiya, N. Nishimiya and M. Suzuki, J. Mol. Spectrosc., 2002, 214, 132.

30 M. Macler, M. Erickson, H. Lin and M. C. Heaven, J. Phys. Chem., 1992, 96, 4301.

31 J. O. Clevenger, E. P. Ray, J. Tellinghuisen, X. Zheng and M. C. Heaven, Can. J. Phys., 1994, 72, 1294.

32 R. Li, C. Wei, Q. Sun, E. Sun, M. Jin and H. Xu, J. Quant. Spectrosc. Radiat. Transfer, 2014, 133, 271.

33 R. E. Willis Jr and W. W. Clark III, J. Chem. Phys., 1980, 72, 4946. 